\title{
Myasthenia gravis associated with renal cell carcinoma: a paraneoplastic syndrome or just a coincidence
}

\author{
Yiming Zheng, Jingjing Luo, Haiqiang Jin, Ran Liu, Hongjun Hao* and Feng Gao*(i)
}

\begin{abstract}
Background: Myasthenia gravis (MG) can occur as a paraneoplastic phenomenon associated with thymoma. The association of MG with renal cell carcinoma (RCC) is not clear. Herein, we describe six cases of MG associated with RCC.

Methods: There were 283 patients diagnosed with MG admitted to our hospital from 2014 to 2019. Among them, 6 patients also had RCC. None of them had immune checkpoint inhibitor therapies. We performed a retrospective clinical data collection and follow-up studies of these 6 patients.

Results: These 6 patients with an average MG onset age of $61.3 \pm 13.3$ years, were all positive for anti-acetylcholine receptor antibodies. MG symptoms appeared after RCC resection in 3 cases. RCC was discovered after the onset of MG in 2 cases, and synchronously with MG in 1 case. After nephrectomy, the MG symptoms showed a stable complete remission in 1 case. Among them, four patients met the diagnostic criteria of possible paraneoplastic neurological syndromes.
\end{abstract}

Conclusions: Except for thymoma, patients with MG should pay attention to other tumors including RCC. MG may be a paraneoplastic syndrome of RCC, and further studies are needed to elucidate the relationship.

\section{Background}

Myasthenia gravis (MG), an autoimmune neuromuscular disease caused by autoantibodies mostly against nicotinic acetylcholine receptors (AChRs) in neuromuscular junctions, can occur as a paraneoplastic phenomenon associated with thymoma [1,2]. The association between extrathymic malignancies and MG is an attractive topic. Several cases of extrathymic malignancies complicated with MG have been reported, considering as paraneoplastic syndromes. Most of the reported extrathymic malignancies are lung cancer [3-7]. As far as we known, there is only one report that MG as a paraneoplastic syndrome associated with renal cell carcinoma (RCC) in the

*Correspondence: haohj1963@126.com; gaofh2011@126.com Neurology Department, Peking University First Hospital, No.8 Xishiku Street, Beijing 100034, China medical literature [7]. To date, there has been no more evidence supporting the speculation that association of MG with RCC might be a paraneoplastic syndrome or just a coincidence. Herein, we describe six cases of MG associated with RCC.

\section{Methods}

There were 283 patients diagnosed with MG admitted to our hospital from 2014 to 2019 by searching the electronic medical record system. These patients all fulfilled the diagnostic criteria for MG. These patients were including 127 males and 156 females (Male to female, 1:1.2), with a mean age of $58.2 \pm 17.2$ years (16-90 years) and an average MG onset age of $54.9 \pm 18.6$ years (4-89 years). The MGFA clinical classification classes were including type I (50 patients), type IIa (31 patients), type IIb (16 patients), type IIIa (30 patients), type IIIb (80 
patients), type IVa (13 patients), type IVb (34 patients) and type $\mathrm{V}$ ( 29 patients). There were $23.0 \%$ patients with thymoma. The presence of anti-AChRs antibodies was $60.6 \%$. Among them, 6 patients also had RCC diagnosed by pathological examination. None of them had immune checkpoint inhibitor therapies. We performed a retrospective clinical data collection and follow-up studies of these 6 cases of MG associated with RCC. Written informed consent was obtained from all participants or their legal surrogate. Research Ethics Committee of The First Hospital of Peking University approved this study.

\section{Results}

These 6 patients were including 5 males and 1 female, with an average MG onset age of $61.3 \pm 13.3$ years (3976 years). Among them, 5 patients had the onset of ptosis, and the other one had the onset of weakness of the proximal limbs. The MGFA clinical classification classes were including one each for I, IIa, IIb, and IIIb and two for IVb. Only one patient had a thymoma. They all had anti-AChRs antibodies. The deltoid, abductor digiti minimi and orbicularis oculi muscles were tested for repetitive nerve stimulation tests. Four patients showed a positive result with a significant decrement at low-frequency stimulations, but all were negative at high-frequency stimulations. Single fiber electromyography was not performed in these patients. RCC was discovered 7 years (case 1) and 4 years (case 2) after the onset of MG in two cases. The other three cases in whom MG symptoms appeared after RCC resection for 1 month (case 3), 5 months (case 4 ) and 6 years (case 5 , with bone metastasis). In the remaining one case (case 6), RCC was discovered by chance when the tumor was screened at the onset of MG. After radical nephrectomy, the symptoms of MG showed a stable complete remission in one case. However, the other five patients who were also treated with immunosuppressants had different outcomes (three with partially improved and two remained unchanged). The detail clinical data of these six patients were showed in the Supplementary material and summarized in Table 1. According to the recommended diagnostic criteria for paraneoplastic neurological syndromes proposed by $\mathrm{F}$ Graus et al., case 1 and case 2 did not meet the possible diagnosis of paraneoplastic neurological syndromes. The other four patients (case 3 to case 6) met the diagnostic criteria for possible paraneoplastic neurological syndromes [8].

\section{Discussion}

All these six patients were presented with typical clinical pattern of myasthenic weakness of the ocular muscles, bulbar muscle or proximal limb muscles, and positive for anti-AChRs antibodies. The diagnosis was MG definite in these six patients [1]. All these patients had pathological diagnosis with RCC, most of which were clear cell type.

There are two types of association between RCC and MG in these six cases. One is the occurrence of RCC in MG patients after many years of treatment that can be a coincidental finding (case 1 and case 2). Case 1 and case 2 did not meet the possible diagnosis of paraneoplastic neurological syndromes, because RCC was discovered more than 2 years after the onset of MG [8]. It is difficult to establish a relationship between these two diseases in these cases. Another type is the presentation of RCC within two years of the diagnosis of MG. There are three cases in whom MG symptoms appeared after RCC resection (case 3 for 1 month, case 4 for 5 months, and case 5 with bone metastasis for 6 years). In case 6 , RCC was discovered at the onset of MG. Thus, these four patients (case 3 to case 6) met the diagnostic criteria for possible paraneoplastic neurological syndromes [8]. After radical nephrectomy, the symptoms of MG showed a stable complete remission without the application of immunosuppressants in case 3 , which is more suggestive of a paraneoplastic association [8, 9]. However, other patients who were also treated with immunosuppressants at the same time had different outcomes. It is difficult to judge whether MG is a paraneoplastic syndrome of RCC through the prognosis in these patients. Because some patients with thymoma-associated MG can only get partial improvement or remain unchanged after thymectomy who need further pharmacological treatment. At the same time, some patients can develop symptoms of MG after thymectomy, just as our case 4 [1, 9].

About 30\% of thymoma patients have MG, while $10-15 \%$ of MG patients have a thymoma [2]. Thymomaassociated myasthenia gravis is a paraneoplastic disease. Except for RCC, the patient in case 4 also had thymoma. In this case, the relationship between RCC and MG is difficult to establish, because MG may be more possible a paraneoplastic disease of thymoma. It is controversial that simultaneous MG with extrathymic malignancies might be one of the paraneoplastic syndromes. Extrathymic malignancies were reported to be relatively common in patients with $\mathrm{MG}$, especially in the older age group [10]. In our 5/6 patients with RCC without thymoma, the average onset-age of MG was 65.8 (range from 57 to 76). Lymphoid malignancy, breast cancer and lung cancer were the most common extrathymic malignant tumors found in MG patients [10, 11]. Except for lymphoid malignancies, there was no difference in the risk of developing other malignant diseases, between the MG and comparison cohort [11]. The association between RCC and MG was only reported in one case report suggesting a paraneoplastic association [7]. There are some other case reports suggesting MG might be a 


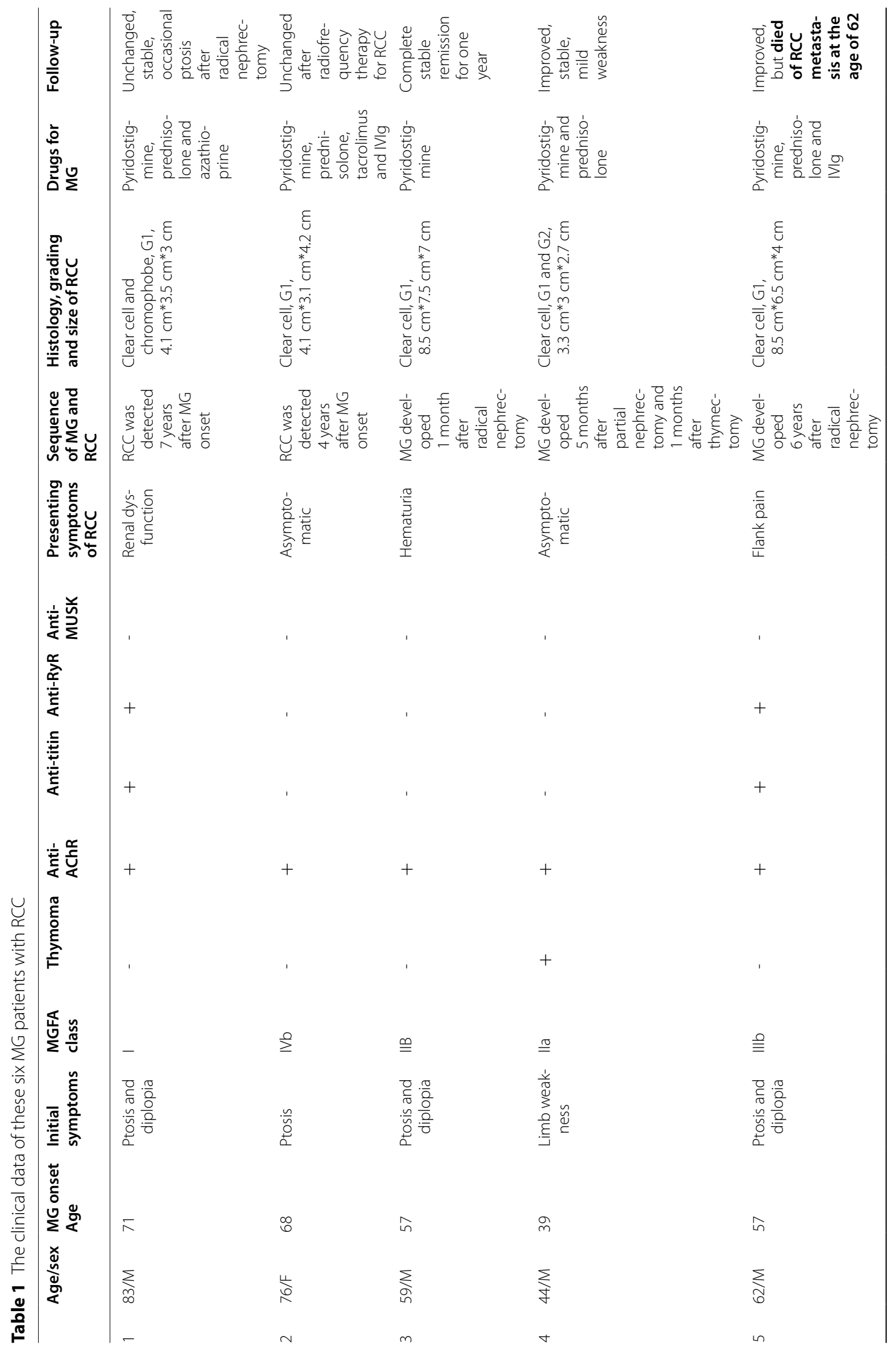




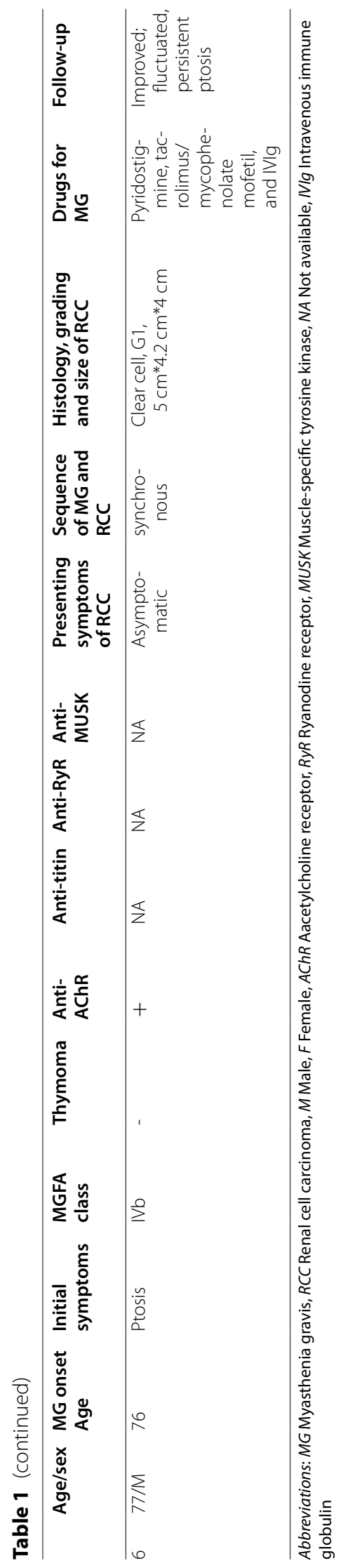


paraneoplastic syndrome of other extrathymic malignancies [3-6]. Furthermore, the incidence of RCC is around $2 \%$ in this cohort of 283 MG patients, which is considerably higher than the incidence reported for the general population worldwide $(6.1 / 100,000$ in male, $3.2 / 100,000$ in female) [12]. Our case series study provides more information of the association between MG and RCC, indicating that physicians should be aware of MG as a potential paraneoplastic syndrome that may be associated with RCC.

RCCs are commonly detected incidentally because of routine ultrasound or CT scans. The classic triad of flank pain, gross hematuria and palpable abdominal mass are found in just $6-10 \%$ of cases [13]. The presenting symptoms of RCC in these six cases including flank pain, gross hematuria and renal dysfunction, while half of them were asymptomatic. Unlike lung cancer, which is easy to find in patients with MG because they usually have chest CT to screen for thymoma $[1,5,6]$. In patients with MG, we need to fully consider the necessity of abdominal imaging to screen for other tumors including RCC.

In RCC, the most common type of paraneoplastic syndrome is the endocrine subtype. Neurological paraneoplastic syndromes are rare events in RCC [14, 15]. The mechanism of neurological paraneoplastic syndromes seems to be immunologic, associated with antibody and $\mathrm{T}$ cell-mediated response to antigens shared between the tumor and neural tissue. The thymus has two sources of AChRs determinants including myoid cells and medullary thymic epithelial cells that produce only unfolded subunits or fragments of AChRs [16]. Similarly, epithelial cells from the proximal convoluted tubule of the kidney, which have been proposed as the cells of origin for the two most common subtypes of RCC (clear cell RCC and papillary RCC), express functional AChRs [17, 18]. Meanwhile, all these six patients were positive for anti-AChRs antibodies. Thus, a similar mechanism may account for the production of anti-AChR antibody in the patients with RCC.

In conclusion, we described six cases of anti-AChR positive MG associated with RCC. Our cases indicate that MG as a potential paraneoplastic syndrome may be associated with RCC. Normal thymus and older age of MG onset prompt additional neoplastic screening leading to an early diagnosis of a potentially devastating cancer including RCC. A coincidental finding should not be excluded in some MG cases with RCC, further studies are needed to elucidate the relationship between MG and RCC.

\section{Abbreviations}

MG: Myasthenia gravis; AChRs: Acetylcholine receptors; RCC: Renal cell carcinoma.

\section{Supplementary Information}

The online version contains supplementary material available at https://doi. org/10.1186/s12883-021-02311-8.

Additional file 1: Supplementary material.

\section{Acknowledgements}

The authors extend their appreciation to these patients and their family members for their support.

\section{Authors' contributions}

YZ contributed to the drafting, and reporting of the case. JJL, HQJ and RL contributed to revision of the manuscript. FG and $\mathrm{HJH}$ contributed to the concept and revision of the manuscript. All authors have read and approved the final manuscript.

\section{Funding}

The study was supported by Scientific Research Seed Fund of Peking University First Hospital (2018SF033). Funding bodies did not play a role in the collection, analysis, and interpretation of data. Funding bodies did not contribute to the writing of this manuscript.

\section{Availability of data and materials}

The datasets used and analyzed during the current study available from the corresponding author on reasonable request.

\section{Declarations}

\section{Ethics approval and consent to participate}

Written informed consent was obtained from all participants or their legal surrogate. Research Ethics Committee of The First Hospital of Peking University approved this study.

\section{Consent for publication}

All the participants or their legal surrogate gave written consent for their personal or clinical details along with any identifying images to be published in this study.

\section{Competing interests}

The authors declare that they have no competing interests.

Received: 19 December 2020 Accepted: 6 July 2021

Published online: 12 July 2021

\section{References}

1. Gilhus NE, Tzartos S, Evoli A, Palace J, Burns TM, Verschuuren JJGM. Myasthenia gravis. Nat Rev Dis Primers. 2019;5(1):30.

2. Gilhus NE, Verschuuren JJ. Myasthenia gravis: subgroup classification and therapeutic strategies. Lancet Neurol. 2015;14:1023-36.

3. Lu T, Song B, Pu H, et al. Paraneoplastic pemphigus and myasthenia gravis as the first manifestations of a rare case of pancreatic follicular dendritic cell sarcoma: CT findings and review of literature. BMC Gastroenterol. 2019;19(1):92.

4. Lanciano N, Lawson V, Arnold W. Paraneoplastic myasthenia gravis associated with appendiceal mucinous neoplasm (P2.093). Neurology. 2014;82(10 Supplement):P2.093.

5. Shaygannejad V, Ghasemi M, Rajaee Z. Myasthenia gravis as a presenting feature in a patient with lung cancer: a case report. J Res Med Sci. 2011;16(2):229-32.

6. Niimi K, Nagata E, Murata N, et al. Lung cancer associated with seronegative myasthenia gravis. Intern Med. 2015;54(11):1381-4. https://doi.org/ 10.2169/internalmedicine.54.3363.

7. Torgerson EL, Khalili R, Dobkin BH, Reiter RE. Myasthenia gravis as a paraneoplastic syndrome associated with renal cell carcinoma. J Urol. 1999;162(1):154. 
8. Graus F, Delattre JY, Antoine JC, et al. Recommended diagnostic criteria for paraneoplastic neurological syndromes. J Neurol Neurosurg Psychiatry. 2004;75(8):1135-40.

9. Skeie GO, Romi F. Paraneoplastic myasthenia gravis: immunological and clinical aspects. Eur J Neurol. 2008;15(10):1029-33.

10. Levin N, Abramsky O, Lossos A, et al. Extrathymic malignancies in patients with myasthenia gravis. J Neurol Sci. 2005;237:39-43.

11. Yeh JH, Lin CC, Chen YK, Sung FC, Chiu HC, Kao CH. Excessive risk of cancer and in particular lymphoid malignancy in myasthenia gravis patients: a population-based cohort study. Neuromuscul Disord. 2014;24(3):245-9.

12. Sung H, Ferlay J, Siegel RL, Laversanne M, Soerjomataram I, Jemal A, Bray F. Global Cancer Statistics 2020: GLOBOCAN Estimates of Incidence and Mortality Worldwide for 36 Cancers in 185 Countries. CA Cancer J Clin. 2021;71(3):209-49. https://doi.org/10.3322/caac.21660.

13. Lee CT, Katz J, Fearn PA, Russo P. Mode of presentation of renal cell carcinoma provides prognostic information. Urol Oncol. 2002;7(4):135-40.

14. Ding G, Feng C, Song N, Fang Z, Xia G, Jiang H, et al. Paraneoplastic symptoms: cachexia, polycythemia, and hypercalcemia are, respectively, related to vascular endothelial growth factor (VEGF) expression in renal clear cell carcinoma. Urol Oncol Semin Orig Investig. 2013;31:1820-5.

15. Hegemann M, Kroeger N, Stenzl A, et al. Rare and changeable as a chameleon: paraneoplastic syndromes in renal cell carcinoma. World J Urol. 2018;36(6):849-54.

16. Marx A, Porubsky S, Belharazem D, et al. Thymoma related myasthenia gravis in humans and potential animal models. Exp Neurol. 2015;270:55-65.

17. Dizman N, Philip EJ, Pal SK. Genomic profiling in renal cell carcinoma. Nat Rev Nephrol. 2020;16:435-51.

18. Yeboah MM, Xue X, Javdan M, Susin M, Metz CN. Nicotinic acetylcholine receptor expression and regulation in the rat kidney after ischemiareperfusion injury. Am J Physiol Renal Physiol. 2008;295(3):F654-61.

\section{Publisher's Note}

Springer Nature remains neutral with regard to jurisdictional claims in published maps and institutional affiliations.
Ready to submit your research? Choose BMC and benefit from:

- fast, convenient online submission

- thorough peer review by experienced researchers in your field

- rapid publication on acceptance

- support for research data, including large and complex data types

- gold Open Access which fosters wider collaboration and increased citations

- maximum visibility for your research: over $100 \mathrm{M}$ website views per year

At BMC, research is always in progress.

Learn more biomedcentral.com/submissions 